\title{
Construction and External Validation of a Nomogram for Predicting Survival of Intrahepatic Cholangiocarcinoma Patients Without Lymph Node and Distant Metastasis
}

\section{Chuang Jiang}

Sichuan University West China Hospital

\section{Fei Teng}

Sichuan University West China Hospital

\section{Yunyou Tang}

West China School of Medicine: Sichuan University West China Hospital

\section{Ziqi Zhang}

Sichuan University West China Hospital

\section{Yimin Chen}

Zunyi Medical University

Zheyu Chen ( $\nabla$ chenzheyu@scu.edu.cn )

Sichuan University West China Hospital https://orcid.org/0000-0003-4059-4774

\section{Research}

Keywords: Intrahepatic cholangiocarcinoma (ICC), Survival, Nomogram,SEER

Posted Date: August 17th, 2021

DOI: https://doi.org/10.21203/rs.3.rs-789620/v1

License: (c) (i) This work is licensed under a Creative Commons Attribution 4.0 International License. Read Full License 


\section{Abstract}

\section{Background}

The purpose of this study was to construct and external validate a nomogram for predicting overall survival(OS) in intrahepatic cholangiocarcinoma (ICC) patients classified as NOMO according to the $7^{\text {th }}$ edition of American Joint Committee on Cancer (AJCC) TNM staging system.

\section{Methods:}

812 ICC patients without distant and lymph node metastasis between 2011 to 2015 were extracted from the Surveillance, Epidemiology, and End Results (SEER) database, then randomly assigned to the training cohort $(n=648)$ or internal validation cohort $(n=164)$, external validation cohort consisted of 136 ICC patients with NOMO stage treated in West China Hospital of Sichuan University from 2013 to 2015. The precision of the nomogram was validated internally using SEER validation cohort and externally using the patients' data of West China Hospital.

\section{Results :}

The nomogram was established to predict 1-year, 3-year and 5-year OS and the calibration curve showed nomogram prediction performance was in good agreement with the actual results. The $\mathrm{C}$-index of the nomogram was $0.750(95 \% \mathrm{Cl}: 0.731-0.769)$ in the training cohort, and the internal and external validated Cindexes were $0.803(95 \% \mathrm{Cl}: 0.783-0.823)$ and $0.681(95 \% \mathrm{Cl}: 0.524-0.838)$, respectively. In the training, internal and external validation cohort, the 1-year, 3-year and 5-year AUCs were $(0.772,0.809,0.798)$, $(0.896,0.868,0.896)$ and $(0.673,0.786,0.886)$, respectively.

\section{Conclusions}

This nomogram has an excellent predictive effect on the 1-,3-, 5-year OS of ICC patients with stage NOMO and guide the optimal treatment for these type of patients.

\section{Introduction}

Intrahepatic cholangiocarcinoma (ICC) is a highly malignant tumor originating from second-degree and above bile duct branches epithelial cells, accounting for $15 \%$ 20\% of primary liver cancer and about $20 \%$ of cholangiocarcinoma[1, 2].The incidence of ICC is only lower than that of hepatocellular carcinoma in primary liver cancer[3]. Over the past few decades, the morbidity and mortality of ICC has been steadily increasing[4-6].Radical surgical resection is still the only potential curative treatment for early-stage ICC at present[5],and regional lymph node dissection should be performed routinely[1, 7]. However, not all ICC patients without metastasis have the opportunity to undergo radical resection. After preoperative evaluation, some patients can only receive radiofrequency ablation(RFA), transcatheter arterial chemoembolization (TACE), palliative resection, etc. Despite partial hepatectomy, the 5 -year survival rate is only about $30 \%$, and the postoperative recurrence rate is as high as $60 \%-70 \%[8]$. Compared with other subtypes of cholangiocarcinoma, local and intrahepatic recurrence of intrahepatic cholangiocarcinoma is the main 
problem after surgery. Therefore, local and systemic treatment after surgery are theoretically necessary. Systemic chemotherapy based on gemcitabine and cisplatin(GC strategy) is usually recommended for patients with advanced or metastasis ICC who have lost the chance of surgery ,yet the median survival is disappointing to less than a year[5].Therefore, early detection and early treatment are particularly important for ICC.

In 2016, the latest edition of American Joint Committee on Cancer(AJCC) staging system, the 8th edition, was released[9]. Compared with the previous staging system, the concept and content of T staging were updated and improved, including adding tumor size as one of the staging factors. In addition, the staging system also highlights the influence of vascular invasion and the relationship between tumor and surrounding tissue on ICC prognosis, but T staging is still controversial. It has been reported that T staging does not well distinguish all ICC patients with different outcomes. Kang et al.[10] found that overall survival was similar in T2 and T3 patients using the 8th edition TNM staging, and that the 8th edition staging system did not significantly improve prognostic performance compared with the 7th edition(C-index:0.628 vs 0.626 ). Svolverato et al.[11]found that according to the 8th edition of TNM, the risk of death in T3 patients was lower than that in $\mathrm{T} 1 \mathrm{~b}$ and $\mathrm{T} 2$ patients.

Therefore, for ICC patients with NOMO stage whose prognosis is mainly predicted by T stage, developing a survival prediction tool has good clinical application value. To our best knowledge, there is currently no survival prediction facility for NOMO staging ICC been reported, and it would be valuable to establish a model that can well predict the prognosis of early ICC. Since the ability of T stage of the 8th editon AJCC TNM staging system to distinguish prognosis is still controversial and the staging information of patients before 2015 is not available, data were extracted from the SEER database to screen NOMO ICC patients using the 7th edition of the AJCC staging system.

Previous studies have shown that a nomogram is an optimal tool that can be used to quantify the prognosis of tumor development by a variety of predictive factors, which is simple and intuitive to apply, and can provide individualized monitoring and follow-up programs for patients, providing support for clinical decision-making[12, 13].For some cancers, the use of nomograms is even superior to the traditional staging system[14-16].

The aim of this study was to determine the factors affecting the prognosis of ICC patients with NOMO stage, and to construct a prediction nomograph of prognosis, then the prognosis model was internally and externally validated based on data from SEER database and West China Hospital of Sichuan University,respectively.

\section{Methods}

\section{Data Source And Selection}

Clinical data of the the ICC patients(international tumor classification:ICD-0-2/ICD-0-3, histology type code : 8160, and the primary site code: $\mathrm{C22.1)[17]that} \mathrm{formed} \mathrm{the} \mathrm{training} \mathrm{cohort} \mathrm{were} \mathrm{extracted} \mathrm{from} \mathrm{database}$ from the SEER database (http://seer.cancer.gov/), to ensure adequate follow-up time, we collected patient 
data between 2011 to 2015. Patients with distant metastases and lymph node metastases; patients without a pathological diagnosis of ICC; patients with incomplete or missing tumor information were excluded, and patients with incomplete survival time or other co-existing malignancies and serious underlying diseases were also excluded. A total of 812 patients met the inclusion criteria were eventually enrolled in the training cohort,the data collection process is shown in Fig. 1. The clinical data we extracted and analyzed in the analysis were as follows:age, gender; marital status, AJCC staging for tumor ( $\mathrm{T}$ ), lymph nodes (N), and presence of metastasis (M); tumor size,tumor number, tumor grade, chemotherapy, radiation, surgery, months of survival.

We applied the 7th edition of the AJCC staging system, which was available for patients between 2004 and 2015. To evaluate the general applicability of the nomogram based on the SEER database for predicting prognosis, we performed external validation using clinical data of 136 patients from West China Hospital of Sichuan University, this study was approved by the ethics committee of our hospital and informed consent of all validation cohort patients and their relatives. The SEER database is publicly available, the basic information of the patients included in the training cohort has been unlabeled, so informed consent was waived.

\section{Statistical Analysis}

Statistical analyses were achieved by R software(version 3.6.1, http://www.r-project.org/) and SPSS Statistics (version 23.0; IBM Corporation). Continuous variables were described as medians with interquartile ranges (IQRs), and frequencies and percentages were used for statistical description of count and grade data. Comparison of categorical variables were performed by the Pearson's chi-square test or the Fisher's exact test. Student's t-test or the Wilcoxon signed-rank test were used to compare continuous variables. We set OS as the main prognostic indicator of ICC patients. OS was defined as the time from diagnosis of ICC to death or final follow-up. Cox proportional hazards regression model was used for univariate analyses to evaluate predictors of long-term prognosis, variables with $p<0.1$ were further analyzed by multivariate analyses. Hazard ratios (HRs) were showed with $95 \% \mathrm{Cl}$. By using the independent risk factors, a nomogram was developed to predict the 1-,3-,5-year OS rates. Harrell's concordance index(Cindex), calibration plot and area under the receiver operating characteristic (ROC) curve (AUC) were used to evaluate the discriminant ability of the nomogram. The values of C-index and AUC are between 0.5 and 1.0, and the higher the value, the stronger the distinguishing ability of the prediction model will be. The cutoff

values of the total points in the nomogram were identified according the maximum of the Youden index, and patients in the training cohort and the validation cohorts were divided into high-risk and low-risk groups by cutoff values. Kaplan-Meier survival curves were analysing using the log-rank test.

Bilateral tests were used for all statistical tests, and a P-value $<0.05$ was considered statistically significant.

\section{Results}

\section{Patients Baseline Characteristics}


A total of 812 eligible patients with a diagnosis of ICC in the SEER database from 2011 to 2015 were randomly enrolled in the training cohort $(n=648)$ and internal validaiton cohort $(n=164)$, in addition, 136 cases of ICC patients from the West China Hospital of Sichuan University from 2013 to 2015 were enrolled as a external validation cohort. The clinical characteristics of patients in these three cohorts were summarized in Table 1. In the training cohort, 333(51.4\%)patients were aged over 65 years and 321(49.5\%) were male, 208(32.1\%) patients underwent resection; in the internal and external validation cohort, $86(52.4 \%)$ and $63(46.3 \%)$ patients were aged over 65 years, 83(50.6\%) and 88(64.7\%) were male, 61(37.2\%)and 53(39.0\%)patients underwent resection, respecitvely. 
Table 1

Baseline clinical characteristics of training cohort, internal validation cohort and external validation cohort.

\begin{tabular}{|c|c|c|c|c|c|c|c|}
\hline \multicolumn{2}{|l|}{ Characteristic } & \multirow[t]{3}{*}{$\begin{array}{l}\mathrm{N} \\
\text { (total) }\end{array}$} & \multicolumn{2}{|l|}{ Patients } & \multicolumn{2}{|l|}{$\begin{array}{l}p \\
\text { value }\end{array}$} & \multirow[t]{3}{*}{$\begin{array}{l}p \\
\text { value }\end{array}$} \\
\hline & & & \multirow{2}{*}{$\begin{array}{l}\text { Training } \\
\text { cohort } \\
\mathrm{n}(\%)\end{array}$} & \multicolumn{2}{|l|}{$\begin{array}{l}\text { Internal } \\
\text { Validation } \\
\text { cohort }\end{array}$} & $\begin{array}{l}\text { External } \\
\text { Validation } \\
\text { cohort }\end{array}$ & \\
\hline & & & & $n(\%)$ & & $\mathrm{n}(\%)$ & \\
\hline Total & & 948 & 648 & 164 & & 136 & \\
\hline \multirow[t]{2}{*}{ Age (years) } & $\leq 65$ & 466 & $315(48.6 \%)$ & 78(47.6\%) & 0.752 & 73(53.7\%) & 0.457 \\
\hline & $>65$ & 482 & $333(51.4 \%)$ & $86(52.4 \%)$ & & $63(46.3 \%)$ & \\
\hline \multirow[t]{2}{*}{ Gender } & Male & 492 & $321(49.5 \%)$ & $83(50.6 \%)$ & 0.273 & $88(64.7 \%)$ & $0.001 *$ \\
\hline & Female & 456 & $327(50.5 \%)$ & $81(49.4 \%)$ & & $48(35.3 \%)$ & \\
\hline \multirow[t]{3}{*}{ Maritalstatus } & Married & 572 & $379(58.5 \%)$ & $106(64.6 \%)$ & 0.634 & $87(64.0 \%)$ & 0.478 \\
\hline & Divorce & 121 & $88(13.6 \%)$ & 18(11.0\%) & & $15(11.0 \%)$ & \\
\hline & Other & 255 & 181(27.9\%) & $40(24.4 \%)$ & & $34(25.0 \%)$ & \\
\hline \multirow[t]{2}{*}{ AJCC T stage } & $1-2$ & 564 & $384(59.3 \%)$ & $95(57.9 \%)$ & 0.504 & $85(62.5 \%)$ & 0.483 \\
\hline & $3-4$ & 384 & $264(40.7 \%)$ & $69(42.1 \%)$ & & $51(37.5 \%)$ & \\
\hline \multirow{3}{*}{$\begin{array}{l}\text { Tumor size } \\
(\mathrm{cm})\end{array}$} & $<5$ & 327 & $213(32.9 \%)$ & $59(36.0 \%)$ & 0.99 & $55(40.4 \%)$ & 0.054 \\
\hline & $5-10$ & 324 & $227(35.0 \%)$ & $46(28.0 \%)$ & & $51(37.5 \%)$ & \\
\hline & $>10$ & 297 & $208(32.1 \%)$ & $59(36.0 \%)$ & & $30(22.1 \%)$ & \\
\hline \multirow[t]{4}{*}{ Tumor grade } & I & 63 & $38(5.9 \%)$ & $11(6.7 \%)$ & 0.506 & $14(10.3 \%)$ & 0.181 \\
\hline & II & 235 & $152(23.4 \%)$ & $49(29.9 \%)$ & & $34(25.0 \%)$ & \\
\hline & III-IV & 194 & 128(19.8\%) & $37(22.5 \%)$ & & $29(21.3 \%)$ & \\
\hline & Unknown & 456 & $330(50.9 \%)$ & $67(40.9 \%)$ & & $59(43.4 \%)$ & \\
\hline \multirow{2}{*}{$\begin{array}{l}\text { Tumor } \\
\text { number }\end{array}$} & 1 & 896 & 608(93.8\%) & 160(97.6\%) & 0.99 & $128(94.1 \%)$ & 0.896 \\
\hline & $>1$ & 52 & $40(6.2 \%)$ & $4(2.4 \%)$ & & $8(5.9 \%)$ & \\
\hline \multirow[t]{3}{*}{ Surgery } & Resection & 322 & 208(32.1\%) & $61(37.2 \%)$ & 0.653 & $53(39.0 \%)$ & 0.128 \\
\hline & $\begin{array}{l}\text { Partial } \\
\text { destruction }\end{array}$ & 40 & $25(3.9 \%)$ & $7(4.3 \%)$ & & $8(5.9 \%)$ & \\
\hline & None & 586 & $415(64.0 \%)$ & $96(58.5 \%)$ & & 75(55.1\%) & \\
\hline
\end{tabular}




\begin{tabular}{|c|c|c|c|c|c|c|c|}
\hline \multirow[t]{2}{*}{ Characteristic } & & \multirow[t]{2}{*}{$\begin{array}{l}\mathrm{N} \\
\text { (total) }\end{array}$} & \multicolumn{2}{|l|}{ Patients } & \multicolumn{2}{|l|}{$\begin{array}{l}\mathrm{p} \\
\text { value }\end{array}$} & \multirow[t]{2}{*}{$\begin{array}{l}\mathrm{p} \\
\text { value }\end{array}$} \\
\hline & & & $\begin{array}{l}\text { Training } \\
\text { cohort } \\
\mathrm{n}(\%)\end{array}$ & $\begin{array}{l}\text { Internal } \\
\text { Validation } \\
\text { cohort } \\
\mathrm{n}(\%)\end{array}$ & & $\begin{array}{l}\text { External } \\
\text { Validation } \\
\text { cohort } \\
\mathrm{n}(\%)\end{array}$ & \\
\hline \multirow[t]{2}{*}{ Chemotherapy } & yes & 437 & $294(45.4 \%)$ & $76(46.3 \%)$ & 0.390 & $67(49.3 \%)$ & 0.408 \\
\hline & no/unknown & 511 & $354(54.6 \%)$ & $88(53.7 \%)$ & & $69(50.7 \%)$ & \\
\hline \multirow[t]{2}{*}{ Radiation } & yes & 147 & $111(17.1 \%)$ & $19(11.6 \%)$ & 0.414 & $17(12.5 \%)$ & 0.184 \\
\hline & no/unknown & 801 & $537(82.9 \%)$ & $145(88.4 \%)$ & & $119(87.5 \%)$ & \\
\hline
\end{tabular}

\section{Independent Risk Factors Of The Training Cohort}

Univariate analysis showed that age, AJCC T stage, tumor size, tumor grade, tumor number, surgery ,maritalstatus, were independent factors affecting $\mathrm{OS}(\mathrm{p}<0.05)$, gender and radiation had no statistical significance on $O S(p>0.05)$. Then, multivariable cox regression analysis was carried out and the final results showed 5 factors with statistical significance:T stage(T3-T4:HR $=1.569,95 \% \mathrm{Cl}: 1.302-1.892, \mathrm{p}<$ 0.001 ), tumorsize $(5-10 \mathrm{~cm}: \mathrm{HR}=1.18,95 \% \mathrm{Cl}: 0.938-1.485, \mathrm{p}=0.065 ;>10 \mathrm{~cm}: \mathrm{HR}=1.229,95 \% \mathrm{Cl}: 0.972-$ $1.554, p=0.043$ ), tumor grade(grade II: $H R=1.052,95 \% \mathrm{Cl}: 0.662-1.672, p=0.830$; grade III-IV: $H R=1.595$, $95 \% \mathrm{Cl}: 1.005-2.531 ; \mathrm{p}=0.048$, grade unknown: $\mathrm{HR}=1.520,95 \% \mathrm{Cl}: 0.980-2.356 ; \mathrm{p}=0.062$ ), surgery (partial destruction: $\mathrm{HR}=0.254,95 \% \mathrm{Cl}: 0.148-0.434, \mathrm{p}<0.001$, resection: $\mathrm{HR}=0.224,95 \% \mathrm{Cl}: 0.172-0.293, \mathrm{p}<$ $0.001)$, chemotherapy $(\mathrm{HR}=0.629,95 \% \mathrm{Cl}: 0.516-0.767, \mathrm{p}<0.001)$, the results of univariate and multivariate regression analysis are shown in Table 2.

Table 2: Results of univariate and multivariate Cox regression analysis of OS on training cohort. 


\begin{tabular}{|c|c|c|c|c|}
\hline \multirow[t]{2}{*}{ Characteristic } & \multicolumn{2}{|l|}{ Univariate analysis } & \multicolumn{2}{|l|}{ Multivariate analysis } \\
\hline & $\mathrm{HR}(95 \% \mathrm{Cl})$ & $\mathrm{p}$ value & $\mathrm{HR}(95 \% \mathrm{Cl})$ & $\mathrm{p}$ value \\
\hline \multicolumn{5}{|l|}{ Age (years) } \\
\hline$\leq 65$ & Reference & & Reference & \\
\hline$>65$ & $1.401(1.175-1.670)$ & $<0.001^{\star *}$ & $1.121(0.930-1.354)$ & 0.231 \\
\hline \multicolumn{5}{|l|}{ Gender } \\
\hline Male & Reference & & & \\
\hline Female & $0.931(0.782-1.109)$ & 0.422 & & \\
\hline \multicolumn{5}{|l|}{ Maritalstatus } \\
\hline Other & Reference & & Reference & \\
\hline Married & $0.792(0.650-0.965)$ & $0.020 *$ & $0.978(0.793-1.207)$ & 0.839 \\
\hline Divorce & $0.860(0.644-1.149)$ & 0.309 & $0.899(0.667-1.213)$ & 0.487 \\
\hline \multicolumn{5}{|l|}{ T stage } \\
\hline T1-T2 & Reference & & Reference & \\
\hline T3-T4 & $1.705(1.428-2.035)$ & $<0.001$ ** & $1.569(1.302-1.892)$ & $<0.001^{\star *}$ \\
\hline \multicolumn{5}{|l|}{ Tumor size (cm) } \\
\hline$<5$ & Reference & & Reference & \\
\hline $5-10$ & $1.363(1.094-1.697)$ & $0.006^{*}$ & $1.18(0.938-1.485)$ & 0.065 \\
\hline$>10$ & $1.992(1.598-2.483)$ & $<0.001^{\star *}$ & $1.229(0.972-1.554)$ & 0.043 \\
\hline \multicolumn{5}{|l|}{ Tumor grade } \\
\hline I & Reference & & Reference & \\
\hline II & $0.955(0.605-1.508)$ & 0.844 & $1.052(0.662-1.672)$ & 0.830 \\
\hline III-IV & $1.624(1.031-2.558)$ & $0.036^{*}$ & $1.595(1.005-2.531)$ & $0.048^{*}$ \\
\hline Unknown & $2.547(1.663-3.899)$ & $<0.001$ ** & $1.520(0.980-2.356)$ & 0.062 \\
\hline \multicolumn{5}{|l|}{ Tumor number } \\
\hline 1 & Reference & & Reference & \\
\hline$>1$ & $1.797(1.202-2.687)$ & $0.004^{*}$ & $1.384(0.918-2.086)$ & 0.120 \\
\hline \multicolumn{5}{|l|}{ Surgery } \\
\hline None & Reference & & Reference & \\
\hline Partial destruction & $0.272(0.162-0.456)$ & $<0.001^{\star *}$ & $0.254(0.148-0.434)$ & $<0.001^{\text {** }}$ \\
\hline
\end{tabular}




\begin{tabular}{|lllll|}
\hline Resection & $0.210(0.168-0.263)$ & $<0.001^{\star \star}$ & $0.224(0.172-0.293)$ & $<0.001^{\star \star}$ \\
\hline Chemotherapy & & & & \\
\hline no/unknown & Reference & & Reference & \\
\hline yes & $0.888(0.745-1.059)$ & 0.094 & $0.629(0.516-0.767)$ & $<0.001^{\star \star}$ \\
\hline Radiation & & & & \\
\hline yes & Reference & & & \\
\hline no/unknown & $0.827(0.654-1.044)$ & 0.11 & & \\
\hline
\end{tabular}

\section{Constructing Prognostic Nomograms For Os}

Based on the significant independent risk factors of multivariate analysis, a nomogram was constructed to predict the 1-,3-,5-year OS of NOMO stage ICC patients(Fig. 2). The nomogram shows that the most affected factor for the OS is the surgery, followed by chemotherapy, AJCC T stage, tumor grade and tumor size. The total points is calculated by adding up the points corresponding to each factor, then locating it on the bottom scale to help clinicians predict 1-,3-,5-year OS for each independent NOMO stage ICC patient. A higher total points reflects a poorer prognosis.

\section{Discrimination And Calibration Of The Nomogram}

In the training cohort, internal and external validation cohort, the calibration plots of the OS predicting nomogram all show good agreement between the predictions and the actual observations of the 1-, 3-, and 5-year OS.(Fig. 3A-I)

The C-index values for OS prediction were $0.750(95 \% \mathrm{Cl}: 0.731-0.769)$ in the training cohort, $0.803(95 \%$ $\mathrm{Cl}: 0.783-0.823)$ in the internal validation cohort, and $0.681(95 \% \mathrm{Cl}: 0.524-0.838)$ in the external validation cohort, which revealed a good prognostic accuracy of the nomogram. We plotted the ROC curves for all cohorts, the AUC values of 1 - 3- and 5-year OS in the training cohort were $0.772,0.809,0.798$, respectively; $0.896,0.868,0.896$ in the internal validation cohort, respectively, and $0.673,0.786,0.886$ in the external validation cohort, respectively, which reveals a nice discriminated ability of the normogram(Fig. 4A-I).

\section{Stratification Of Risk Groups}


From the maximum values of the Youden indexes of the ROC curves, we obtained the cutoff values of 110 based on the nomogram. ICC patients in the training cohort, the internal and external validation cohort were then divided into high-risk(total points $\geq 110$ ) and low-risk(total points $<110$ ) groups respectively. The Kaplan-Meier survival curves of the three cohorts were drawn, and the curves all showed that patients in the low-risk group obtained significantly better OS than patients in the high-risk group. (Fig. 5A-C).

\section{Discussion}

Intrahepatic cholangiocarcinoma is a type of primary liver cancer originating from intrahepatic bile duct epithelial cells with poor prognosis and lack of effective treatment. The incidence of intrahepatic cholangiocarcinoma has been increasing worldwide in the last 30 years[18]. For some patients of early stage ICC, surgical treatment has been recognized as the most effective treatment to improve the $0 S[5$, 19].Due to the insidious and invasive characteristics of ICC, it is often not diagnosed in time. Many ICC patients already metastasis at the time of initial diagnosis, losing the chance of surgery always means a bad prognosis.

Nomogram is a intuitive, and effective graphical math model developed based on independent prognostic factors of cancer, it is used for survival prediction of various cancers[20-22]. A good nomogram can predict prognosis even better than traditional TNM staging systems $[15,16]$.

In our study, based on the SEER database, we constructed and internally validated a nomogram to predict the OS at 1-, 3- and 5-years of ICC patients with stage NOMO. External validation was performed using a validation cohort composed of inluded ICC patients from West China Hospital(C-index:0.681,95\% Cl:0.5240.838). To our knowledge, this is the first prognostic tool specifically for NOMO-stage ICC patients, five indicators(surgery, chemotherapy, AJCC T stage, tumor grade, tumor size ) are included in this nomogram. Consistent with other studies on ICC for different purposes[23, 24], we find that surgery is the most important factor affecting OS among the 5 indicators in the nomogram, meanwhile, it reveals that chemotherapy can also improve the prognosis of ICC patients, which is consistent with previous studies that reported that surgery combined with adjuvant chemotherapy has better efficacy than surgery alone[25-27].

The staging system of ICC is constantly being updated and properly modified but the effect of tumor size on the prognosis of ICC patients has been controversial, prior to the 7th edition of the AJCC staging system, tumor diameter was not considered as a staging factor[28]. Although there are some differences in selected cut-off values, tumor size has been reported to be correlated with the prognosis of ICC patients[29-31]. What's more, tumor size has been included in the 8th edition AJCC staging system and based on a large body of evidence-based medical evidence,the tumor diameter of $5 \mathrm{~cm}$ was selected as the cut-off for the T1 stage refinement $(\mathrm{T} 1 \mathrm{a}, \mathrm{T} 1 \mathrm{~b})[9]$, later, some scholars also verified it[32]. Due to the lack of 8th edition AJCC staging system information for ICC patients in the earlier SEER database, and the lack of specific information of the tumor, we could not directly convert from 7th or 6th stage to 8th stage. We used $T$ staging of the 7th AJCC staging system as a variable and included tumor size as a separate predictor and we used $5 \mathrm{~cm}$ and $10 \mathrm{~cm}$ as the cutoff values for classifying tumor size. We found that both tumor size and the AJCC T stage were impact factors of prognosis, patients with larger tumors have a worse prognosis, 
which is consistent with published studies and justifies the inclusion of tumor size as a staging indicator in the 8th edition of the staging system.

This nomogram had some certain advantages, for instance, the training cohort and the internal validation cohort are mainly composed of patients from the United States, while the external validation cohort mainly contains yellow race from China, indicating this nomogram is suitable for predict the prognosis of ICC patients at NOMO stage from different regions and ethnic groups. Then, there was no age limit for patients in our inclusion criteria, all the range of ages meet the criteria were included, not as some other studies just include the young or the old, so the nomogram is suitable for all the ICC patients at NOMO stage. This nomogram does not include age as one of the indicators, because multivariate analysis did not find a significant effect of age on OS in the training group, which may be due to the relatively small effect of age on prognosis due to the significantly shortened survival time of ICC patients, similarly, Horgan et al.[33] found that for patients with biliary tract cancers, there was no significant difference in survival rates between younger and older patients after surgical treatment.

To verify the predictive stability of this nomogram, we performed external validation, as evaluated by the AUCs and c-index values, the nomogram all showed good predictive performance. The validation plots of the 1-, 3-, and 5-year OS predicting nomogram all demonstrated the relatively good accurate between the predictions and the actual observations. This means that this nomogram will be helpful for clinicians' formulation of personalized treatment stratage for specific ICC patients with a NOMO stage.

Although the establishment of the model was based on a large sample size, and the external validation cohort was selected from relatively representative hospitals in China, our study still had some limitations. First, the data analysed in this study were collected retrospectively, and there may be potential bias due to unknown confounding factors. Second, all the variables used in the modeling were selected from the SEER database, but some data that might be related to prognosis were not provided in this database, such as the specific radiotherapy dose and chemotherapy stratage, the specific site of lymph node dissection, tumor marker such as carbohydrate antigen 199(CA19-9), and resection margins. Finally, we excluded some patients with incomplete clinical data, which may also lead to selection bias.

\section{Conclusion}

We developed a nomogram to predict 1-, 3-, and 5-year OS in NOMO stage ICC patients.Using the SEER and the external validation datasets of West China Hospital reveals good resolution and calibration ability. Therefore, this nomogram may help predict individual survival in ICC patients without lymph nodes or distant metastasis and guide clinicians in the development of treatment strategies.

\section{Declarations}

\section{Acknowledgments}

Not Applicable. 


\section{Authors' contributions}

Conception and design: CJ, FT, YYT. Drafted the initial manuscript: CJ. Data collection:CJ,FT,YMC. Analysis and interpretation of the data:FT,ZQZ. Analyses, reviewed and revised the manuscript:ZYC. All authors read and approved the final manuscript.

\section{Funding}

Not Applicable.

\section{Availability of data and materials}

The data that support the findings of this study are available from the corresponding author upon reasonable request.

\section{Ethics approval and consent to participate}

The Ethics Committee of West China Hospital of Sichuan University approved the study and informed consent was obtained from all patients in the validation cohort and their relatives.

\section{Consent for publication}

Not Applicable.

\section{Competing interests}

The authors declare that they have no competing interests.

\section{References}

1. Aloia T. Pawlik TM, Ta Ouli B et al. Intrahepatic Bile Ducts. AJCC Cancer Staging Manual.8th edition. New York: Springer,2017;295-302.

2. Najran P. Lamarca A, Mullan D, et al. Update on Treatment Options for Advanced Bile Duct Tumours: Radioembolisation for Advanced Cholangiocarcinoma. Current oncology reports 2017; 19:50.

3. Rizvi S. Khan S, Hallemeier C, et al. Cholangiocarcinoma - evolving concepts and therapeutic strategies. Nat Rev Clin Oncol 2018; 15:95-111.

4. Rahnemai-Azar A. Weisbrod A, Dillhoff M, et al. 4Intrahepatic cholangiocarcinoma: Molecular markers for diagnosis and prognosis. Surg Oncol 2017; 26:125-37.

5. Bridgewater J. Galle P, Khan S, et al. Guidelines for the diagnosis and management of intrahepatic cholangiocarcinoma. Journal of hepatology $2014 ; 60: 1268-89$. 
6. Fitzmaurice C. Allen C, Barber R, et al. Global, Regional, and National Cancer Incidence, Mortality, Years of Life Lost, Years Lived With Disability, and Disability-Adjusted Life-years for 32 Cancer Groups, 1990 to 2015: A Systematic Analysis for the Global Burden of Disease Study. JAMA oncology 2017; 3:52448.

7. Weber S. Ribero D, O'Reilly E, et al. Intrahepatic cholangiocarcinoma: expert consensus statement. HPB: the official journal of the International Hepato Pancreato Biliary Association 2015; 17:669-80.

8. Chun Y. Javle M. Systemic and Adjuvant Therapies for Intrahepatic Cholangiocarcinoma. Cancer control: journal of the Moffitt Cancer Center 2017; 24(3):594-9.

9. Amin MB. Greene FL, Edge SB, et al. The Eighth Edition AJCC Cancer Staging Manual: Continuing to build a bridge from a population-based to a more "personalized" approach to cancer staging. CA Cancer J Clin 2017; 67:93 - 9.

10. Kang S. Hwang S, Lee Y, et al. Prognostic comparison of the 7th and 8th editions of the American Joint Committee on Cancer staging system for intrahepatic cholangiocarcinoma. J Hepato-Biliary-Pancreat Sci 2018; 25:240-8.

11. Spolverato G. Bagante F, Weiss M, et al. Comparative performances of the 7th and the 8th editions of the American Joint Committee on Cancer staging systems for intrahepatic cholangiocarcinoma. Journal of surgical oncology 2017; 115:696-703.

12. Zeng Y. Mayne N, Yang C, et al. A Nomogram for Predicting Cancer-Specific Survival of TNM 8th Edition Stage I Non-small-cell Lung Cancer. Ann Surg Oncol 2019; 26:2053-62.

13. Strobel O. Büchler M. [A nomogram for the prediction of malignancy in branch-duct IPMN]. Der Chirurg; Zeitschrift fur alle Gebiete der operativen Medizen 2019; 90:92..

14. Wang Y. Li J, Xia Y, et al. Prognostic nomogram for intrahepatic cholangiocarcinoma after partial hepatectomy. Journal of clinical oncology: official journal of the American Society of Clinical Oncology 2013; 31:1188-95.

15. Zhang Y. Wu Z, Wang X, et al. Development and external validation of a nomogram for predicting the effect of tumor size on survival of patients with perihilar cholangiocarcinoma. BMC Cancer 2020; 20:1044.

16. Pan Z. You H, Bu Q, et al. Development and validation of a nomogram for predicting cancer-specific survival in patients with Wilms' tumor. J Cancer 2019; 10:5299-305.

17. Vitale A. Moustafa M, Spolverato G, et al. Defining the possible therapeutic benefit of lymphadenectomy among patients undergoing hepatic resection for intrahepatic cholangiocarcinoma. Journal of surgical oncology 2016; 113:685-91.

18. Sirica A. Gores G, Groopman J, et al. Intrahepatic Cholangiocarcinoma: Continuing Challenges and Translational Advances. Hepatology 2019; 69:1803-15.

19. Groot Koerkamp B. Jarnagin W. Surgery for perihilar cholangiocarcinoma. Br J Surg 2018; 105:771-2.

20. Xiao Z. Shi Z, Hu L, et al. A new nomogram from the SEER database for predicting the prognosis of gallbladder cancer patients after surgery. Annals of translational medicine 2019; 7:738. 
21. Li J. Liu L. Overall survival in patients over 40 years old with surgically resected pancreatic carcinoma: a SEER-based nomogram analysis. BMC Cancer 2019; 19:726.

22. Wang S. Liu Y, Shi Y, et al. Development and external validation of a nomogram predicting overall survival after curative resection of colon cancer. J Int Med Res 2021; 49:3000605211015023.

23. Yuan C. Hu Z, Wang K. Zou S. Development and Validation a Nomogram for Predicting Overall Survival in Patients With Intrahepatic Cholangiocarcinoma. Frontiers in surgery 2021; 8:659422.

24. Zhu H. Ji K, Wu W, et al. Describing Treatment Patterns for Elderly Patients with Intrahepatic Cholangiocarcinoma and Predicting Prognosis by a Validated Model: A Population-Based Study. J Cancer 2021; 12:3114-25.

25. Schweitzer N. Weber T, Kirstein M, et al. 31The effect of adjuvant chemotherapy in patients with intrahepatic cholangiocarcinoma: a matched pair analysis. J Cancer Res Clin Oncol 2017; 143:134755 .

26. Miura J. Johnston F, Tsai S, et al. Chemotherapy for Surgically Resected Intrahepatic Cholangiocarcinoma. Ann Surg Oncol 2015; 22:3716-23.

27. Reames B. Bagante F, Ejaz A, et al. Impact of adjuvant chemotherapy on survival in patients with intrahepatic cholangiocarcinoma: a multi-institutional analysis. HPB: the official journal of the International Hepato Pancreato Biliary Association 2017; 19:901-9.

28. Edge S. Compton C. The American Joint Committee on Cancer: the 7th edition of the AJCC cancer staging manual and the future of TNM. Ann Surg Oncol 2010; 17:1471-4.

29. Nathan H. Aloia T, Vauthey J, et al. A proposed staging system for intrahepatic cholangiocarcinoma. Ann Surg Oncol 2009; 16:14-22.

30. Sakamoto Y. Kokudo N, Matsuyama Y, et al. Proposal of a new staging system for intrahepatic cholangiocarcinoma: Analysis of surgical patients from a nationwide survey of the Liver Cancer Study Group of Japan. Cancer 2016; 122:61-70.

31. Kong J. Cao Y, Chai J, et al. Effect of Tumor Size on Long-Term Survival After Resection for Solitary Intrahepatic Cholangiocarcinoma. Frontiers in oncology 2020; 10:559911.

32. RuiYang W. ZhiMing Y, Jiao F, et al. Evaluation and Recommendation of the 8th Edition of American Joint Committee on Cancer (AJCC) Staging System for Intrahepatic Cholangiocarcinoma (ICC) in 820 Patients from the Surveillance, Epidemiology, and End Results (SEER) Database. Journal of gastrointestinal surgery: official journal of the Society for Surgery of the Alimentary Tract 2021; 25:145-54.

33. Horgan A. Knox J, Aneja P, et al. Patterns of care and treatment outcomes in older patients with biliary tract cancer. Oncotarget 2015; 6:44995-5004.

\section{Figures}




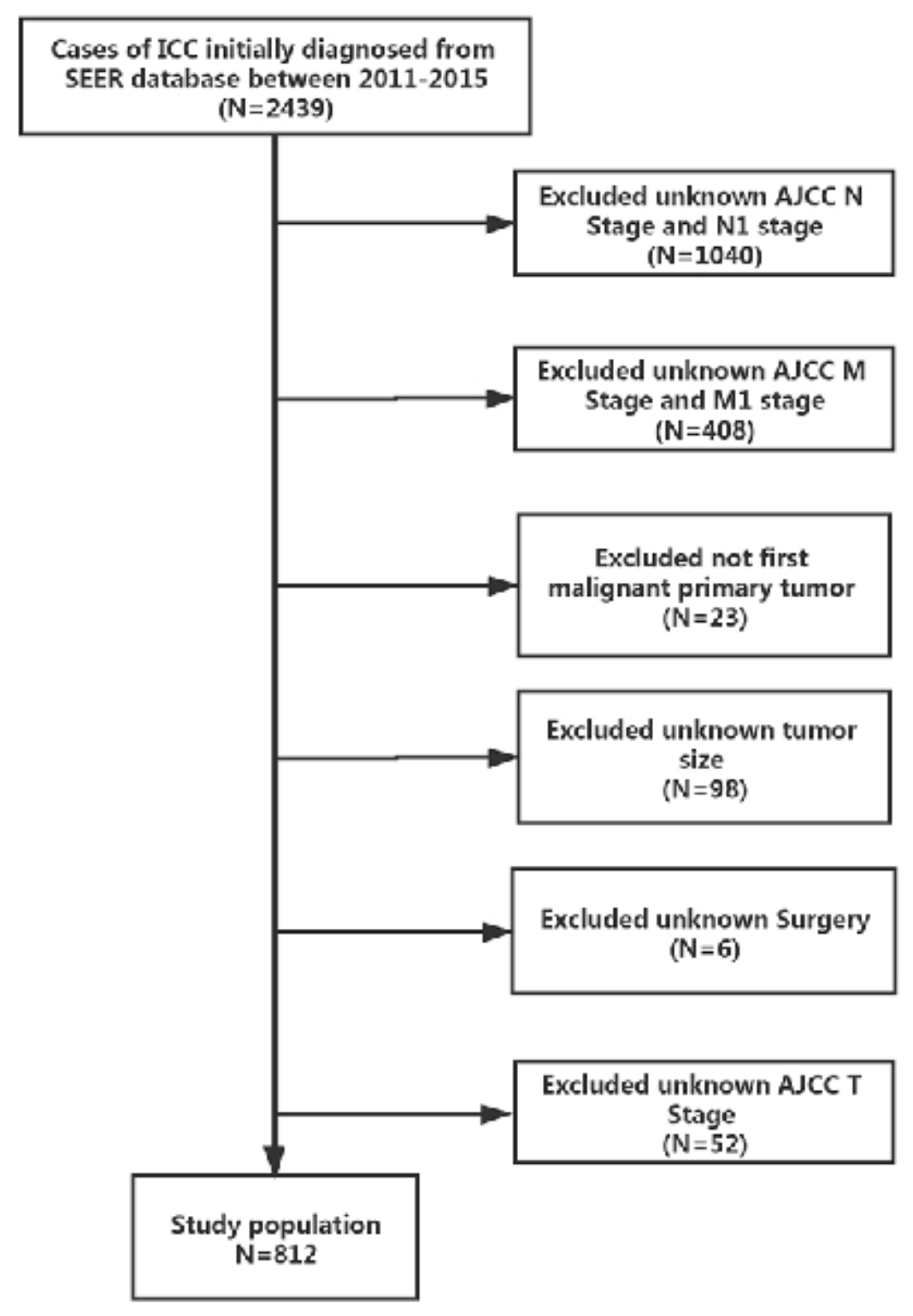

Figure 1

The flow diagram of the screening process for the target ICC patient population from the Surveillance, Epidemiology, and End Results (SEER) database. 
Points

Surgery

$\mathrm{T}$

Grade

Chemotherapy

Tumorsize

Total Points

1-Year Survival

3-Year Survival

5 -Year survival

$\begin{array}{lllllllllll}0 & 10 & 20 & 30 & 40 & 50 & 60 & 70 & 80 & 90 & 100\end{array}$

Partial destruction

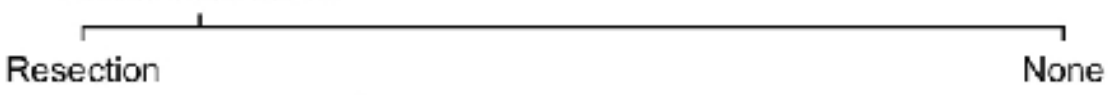

T3-T4

$\mathrm{T} 1-\mathrm{T} 2$

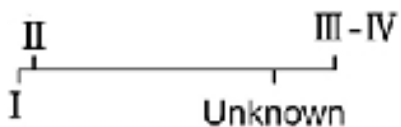

nolunknown

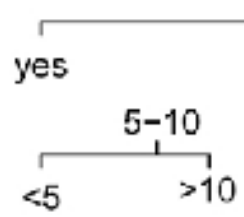

$\begin{array}{lllllllllll}0 & 20 & 40 & 60 & 80 & 100 & 140 & 180 & 220\end{array}$

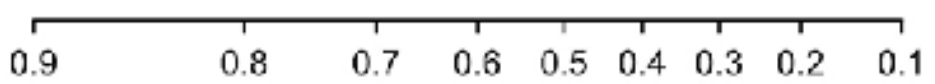

\begin{tabular}{lllllll}
\hline 0.7 & 0.6 & 0.5 & 0.4 & 0.3 & 0.2 & 0.1
\end{tabular}

Figure 2

The nomogram for predicting 1-, 3- and 5-year overall survival rates of NOMO stage ICC patients. T:AJCC T stage 

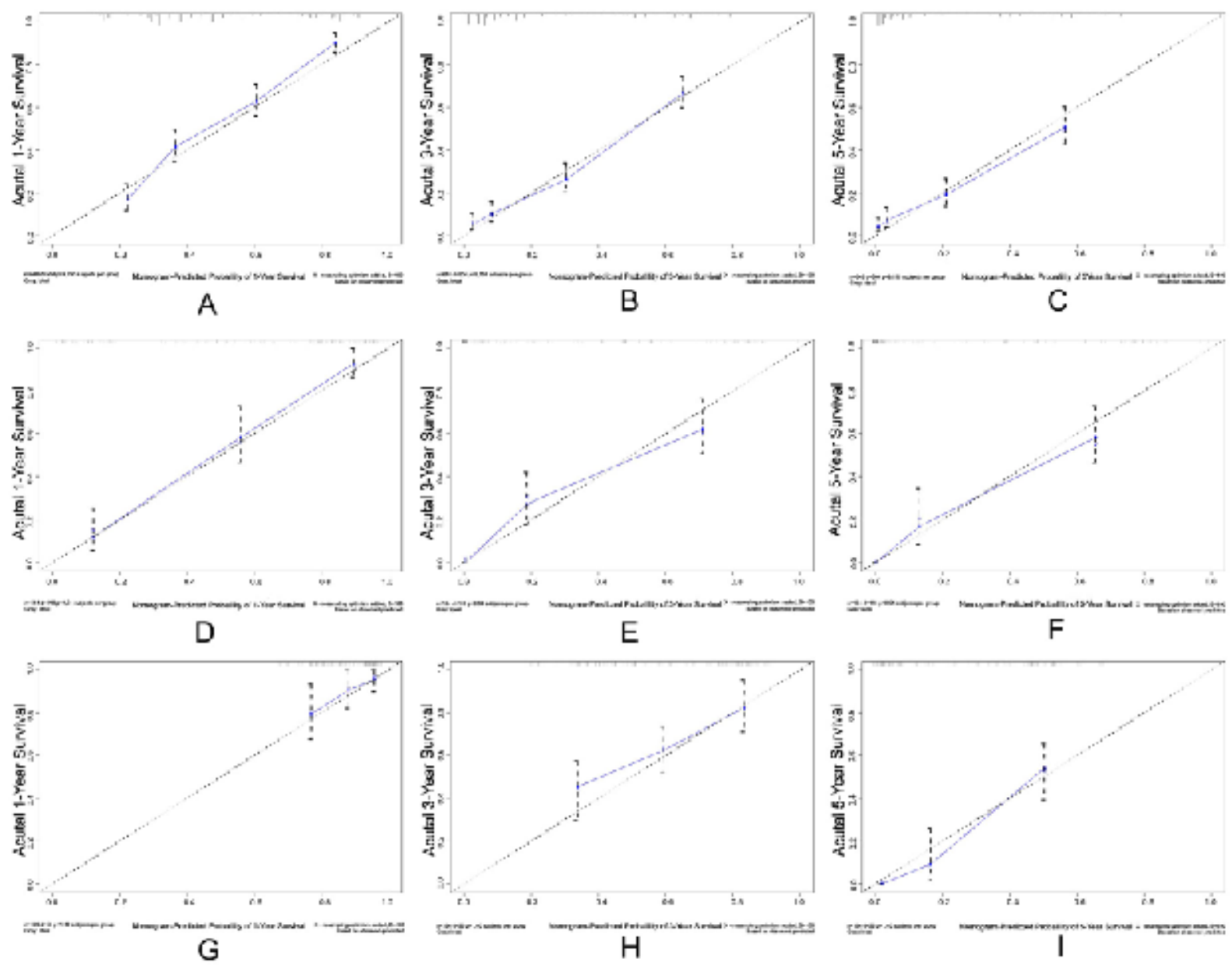

\section{Figure 3}

Calibration plots predicting the 1-, 3- and 5-year OS of patients in the training cohort $(A, B, C)$, internal validation cohort $(\mathrm{D}, \mathrm{E}, \mathrm{F})$ and external validation cohort $(\mathrm{G}, \mathrm{H}, \mathrm{I})$. 


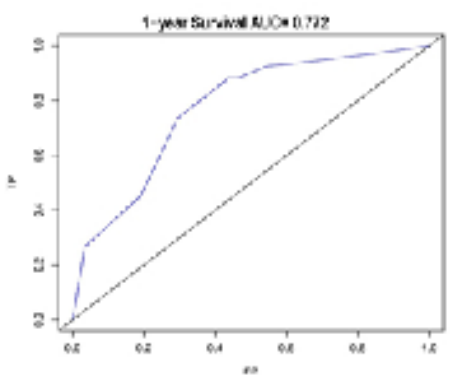

A

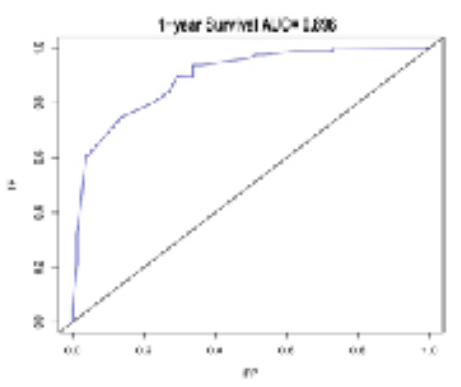

D

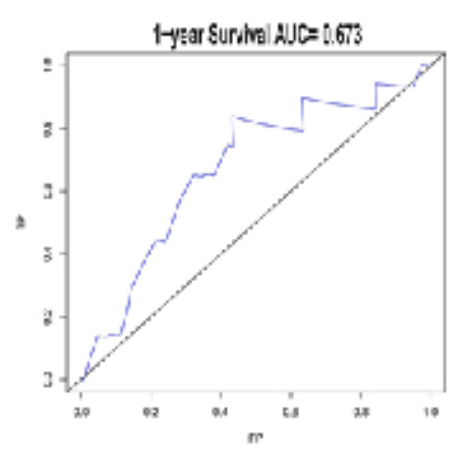

$\mathbf{G}$

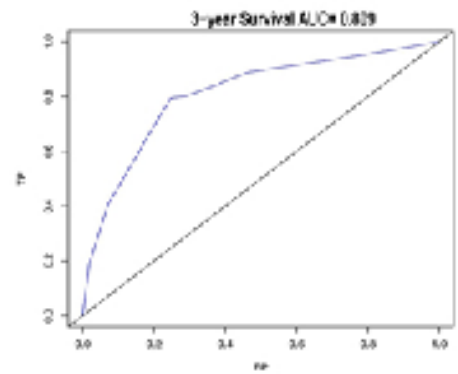

B

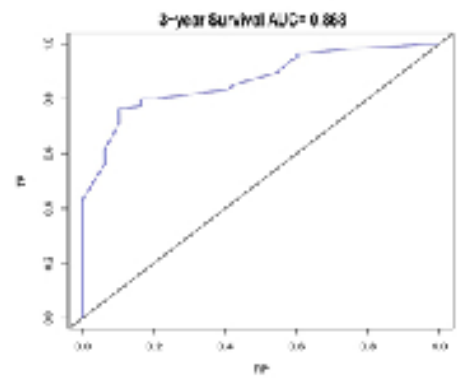

$\mathrm{E}$

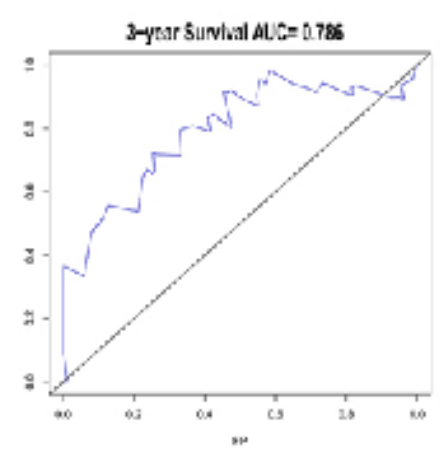

H

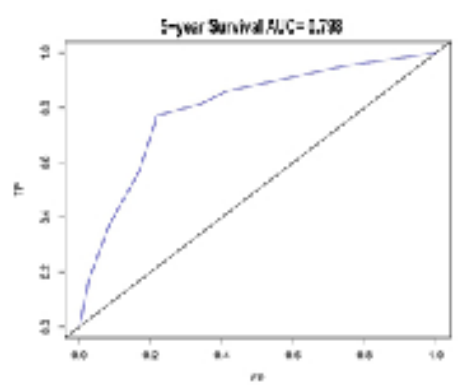

C

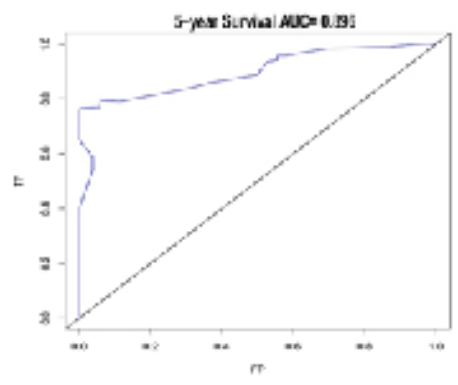

F

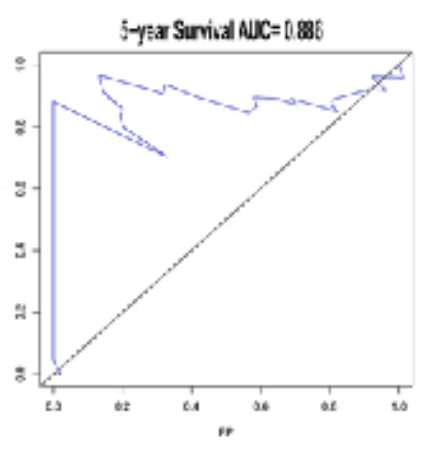

I

\section{Figure 4}

ROC curves and AUCs of 1-, 3- and 5-year OS in training cohort $(A, B, C)$, internal validation cohort $(D, E, F)$ and external validation cohort $(\mathrm{G}, \mathrm{H}, \mathrm{I})$. AUC: area under the curve; ROC: receiver operating characteristic; $\mathrm{TP}$ : true positive rates; FP: false positive rate. 


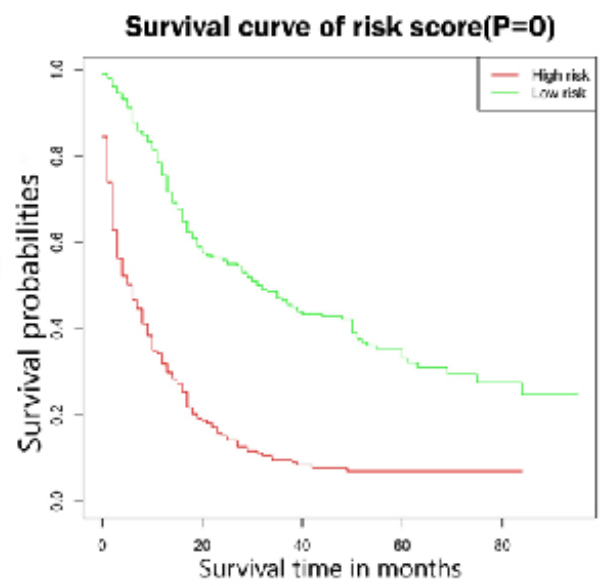

A

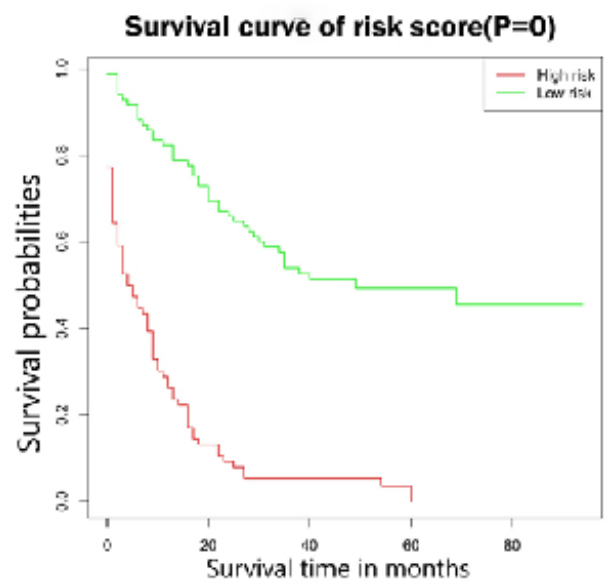

B

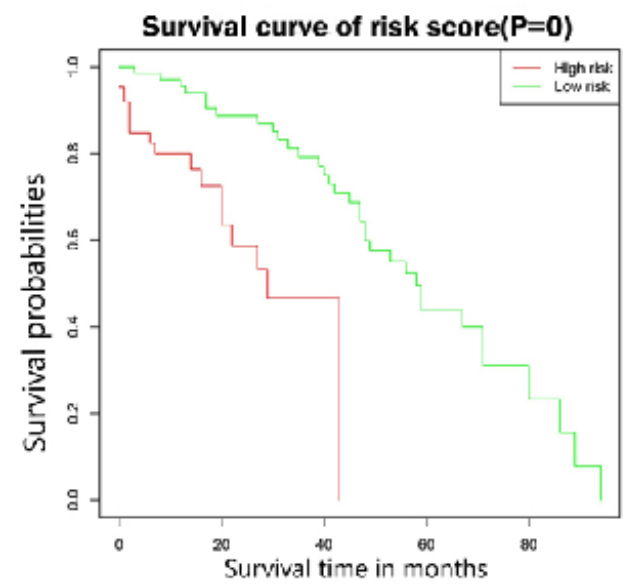

C

\section{Figure 5}

Kaplan-Meier curves of high-risk and low-risk patients in the training cohort(A),internal validation cohort(B) and external validation cohort(C). 